\title{
Safety Performance Evaluations for the Vehicle Based Movable Barriers Using Full Scale Crash Tests
}

\author{
Minsoo Jin ${ }^{1}$, Sukki Lee ${ }^{2}$, Wonil Park ${ }^{2}$ and Insong Park ${ }^{3}$ \\ ${ }^{1}$ Korea Institute of Civil Engineering and Building Technology, and Department of Transportation Engineering, University of Seoul, Korea \\ ${ }^{2}$ Korea Institute of Civil Engineering and Building Technology, Korea \\ ${ }^{3}$ Smart Air Chamber Co., Ltd., Korea
}

\begin{abstract}
The present study aims to develop a prototype of large-size movable barriers to protect roadside workers from incoming vehicles to the road work area with the following functions: maximization of work space in the right and left directions, convenient mobility, and minimization of impact without modification of the inside of movable barriers into traffic lanes and perform safety performance assessment on passengers through full scale crash tests. The large movable barrier was divided into folder type and telescope type and the development stage was now at the prototype phase. A full scale crash test was conducted prior to certification test at a level of $90 \%$. The full scale crash test result showed that both types of folder type movable barrier and telescope type movable barrier satisfied the standard of the passenger safety performance evaluation at a level of $90 \%$.
\end{abstract}

\section{Introduction}

The Highway Safety Manual(HSM, 2010) in the USA suggested that traffic accidents occur due to three factors: human factors(93\%), vehicle factors(34\%) and roadway/environment factors $(13 \%)$ [1].

Due to the education, enforcement of law, and technological development to reduce traffic accidents, the number of traffic accidents has been decreased overall in recent 10 years but a sole-car accident type and accidents during road works have been increased rather, which needs to establish a management and prevention measure

In particular, the number of casualties during road works is 27 out of 583 workers in the regional office in the Ministry of Land, Transport and Maritime who work for maintenance of general national roads as of 2012 . This number means approximately $5 \%$ of the road workers were dead or injured, which was much higher proportion of casualties than that of fire-fighting officers $(0.97 \%)$ classified as one of the high risk groups. The number of traffic safety-re lated accidents of road workers was 114 cases and 152 workers were damaged (10 fatal and 142 injured workers), which were produced due to careless driving (e.g., mobile phones or digital multimed ia broadcasting (DMB) manipulation, forwardlooking neglect, and drowsy driving etc.) while driving on road work sections in general national roads from 2003 to 2012. This result means that one out of four workers $(25 \%)$ has experienced accidents during their average work period (10 years) [2, 3].

For national exp ressways, more workers were dead or injured from 2008 to 2013 due to worse traffic environment against road workers than that of general national roads, which resulted in 56 fatal casualties and 15 injuries of general drivers and 47 deaths and 15 injuries of road workers (103 deaths and 30 injuries in total). That is, about 22 casualties in road works occur in a year.

For general national roads, new employment of road workers is limitedly added so the number of road workers has been decreased gradually from 773 in 2004, 625 in 2008, and 583 in 2013 whereas the road maintenance and repair jobs were not decreased thereby inputting road workers for traffic control to road works due to the insufficient number of road workers [3].

In recent years, much attention has been paid to road maintenance due to the increases in demand on road services and traffic volume and the construction records of road works in Seoul during 2002 to 2004 showed that 320 road works a day have been conducted on average and this trend has been increased. The degradation in inherent functions of road due to road works is closely related to not only traffic flow but also safety problems of road users and has become a significant threat to the safety of road workers particularly [4].

The present study aims to develop a prototype of large-size movable barriers to protect roadside workers from incoming vehicles to the road work area with the following functions: maximization of work space in the right and left directions, convenient mobility, and minimization of impact without modification of the inside of movable barriers into traffic lanes and perform safety performance assessment on passengers through full scale crash tests. 


\section{Review and concept for development}

\subsection{Review}

Caltrans was developed a Balsi Beam trailer system consists of a semi-truck tractor and a trailer in 2003. The trailer has two telescoping expandable steel beams along each side of the trailer that rotate to provide a stackable two beam protection barrier on either side of the trailer. The beams can be rotated to either side depending on the location of the work that beams.

FHWA(Federal Highway Administration) recognized the necessity of road maintenance worker's injury prevention. So FHWA and Mobilebarriers LLC had conducted study of a movable barrier to protect workers in a side collision accidents and developed and operating MBT-1.

The Smart Air Chamber Co., Ltd. developed H-beam type movable protective car based on Article 4 of Automobile Management Act to protect road workers in freeway workzone.

\subsection{Concept for development}

Based on the literatures and website based review, we established the basic concept to develop the movable safety barriers. The first consideration we made is the idea that the type of barriers needs to be varied according to the work zone characteristics. The second is that the barrier should meet the required safety performance designated by Government of Korea.

With the concept, we designed large-sized movable barriers presuming that barriers can be differently applied according to the characteristic of work zone. Large-sized movable barriers are again classified into two types such as Folder Type and Telescope Type according to the kinematic mechanis $m$, and the former type has foldable feature and the latter type has expansion and contraction operative feature.

Figure 1 shows the folder type of movable barrier, which has the series of the block units. While moving, the block unit is folded to meet the vehicle code designated by Government such as the maximum length of the vehicle. When the vehicle begins to service, the folded block is stretched to the certain length to protect the work zone effectively.

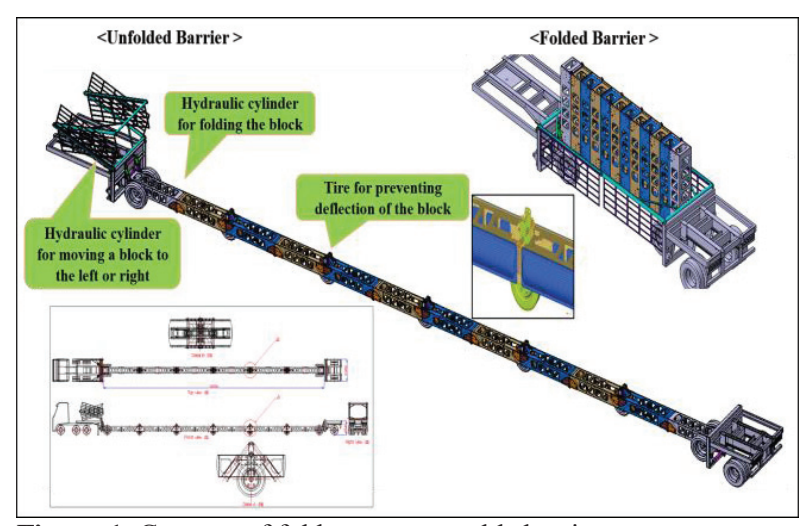

Figure 1. Concept of folder type movable barrier
Figure 2 shows the telescope type of movable barrier, which has the same feature while moving but the barrier beam is expanded from the rear side of the trailer is moving backward [5].

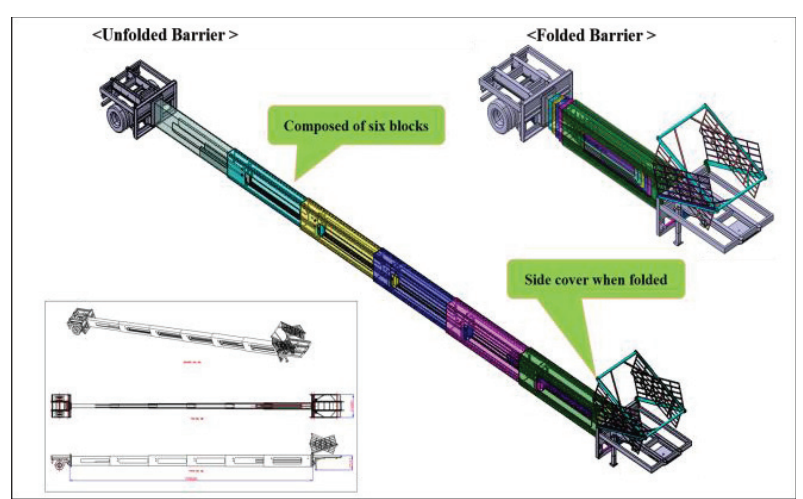

Figure 2. Concept of telescope ty pe movable barrier

\section{Full scale crash tests}

The full-scale crash test of barriers should be conducted in accordance with the "Guideline for Safety Barriers (Safety facility of barriers for vehicles, 2014)" and performance test should be performed by the performance evaluation institution of road safety facility in order to certify the result of performance evaluation. Table 1 presents the test conditions to evaluate the safety of occupants.

Table 1. Test conditions for occupant protections

\begin{tabular}{|c|c|c|c|}
\hline Level & $\begin{array}{c}\text { Impact } \\
\text { Velocity } \\
(\mathrm{km} / \mathrm{h})\end{array}$ & $\begin{array}{c}\text { Impact } \\
\text { Wight(kg) }\end{array}$ & $\begin{array}{c}\text { Impact } \\
\text { Angle(deg.) }\end{array}$ \\
\hline SB1 & 60 & & \\
\cline { 1 - 2 } SB2, SB4 & 80 & \multirow{2}{*}{900} & \multirow{2}{*}{20} \\
\cline { 1 - 2 } SB3 & 100 & & \\
\cline { 1 - 2 } $\begin{array}{c}\text { SB5, SB6, } \\
\text { SB7 }\end{array}$ & 100 & & \\
\cline { 1 - 2 } $\begin{array}{c}\text { SB3-B, } \\
\text { SB5-B }\end{array}$ & 120 & & \\
\hline
\end{tabular}

\subsection{Criteria of occupant protections}

The criteria of occupant protection performance evaluation are presented in Table 2.

Theoretical Head Impact Velocity(THIV) refers to an instantaneous relative velocity between the "theoretical occupant head" and the vehicle when the vehicle is decelerated after crash with facility and the head is free flight thereby crashing to the virtually set surface in the inner space in the vehicle assuming that the occupant head and vehicle are free flight at the impact speed. 
Post-impact Head Deceleration(PHD) refers to the calculated maximum of $10 \mathrm{~m} / \mathrm{s}$ average acceleration of vehicle after THIV is calculated assuming that an occupant maintains a contact with a virtually-set surface inside the vehicle and receives the same acceleration of the vehicle.

Acceleration Severity Index(ASI) refers to a value that represents a sum of each ratio of $50 \mathrm{~ms}$ average acceleration in the $\mathrm{x}, \mathrm{y}$, and $\mathrm{z}$ directions, respectively. It is expressed as a dimensionless scalar. It has a positive value always and if the value is larger than one, a risk of occupant becomes larger.

Table 2. Vehicle occupant impact severity criteria

\begin{tabular}{|c|c|c|}
\hline Criteria & Unit & Index severity \\
\hline THIV & $\mathrm{m} / \mathrm{s}(\mathrm{km} / \mathrm{h})$ & $9(33)$ \\
\hline PHD & $\mathrm{g}$ & 20 \\
\hline ASI & - & 1.9 \\
\hline
\end{tabular}

\subsection{Field setting of crash tests}

A full-scale crash test field was constructed by Smart A ir Chamber Co., Ltd. that conducted the present study with their own investment in Ansung Gyeonggi-do. The test field has been utilized as a full-scale test of front, side, and rear of vehicles.

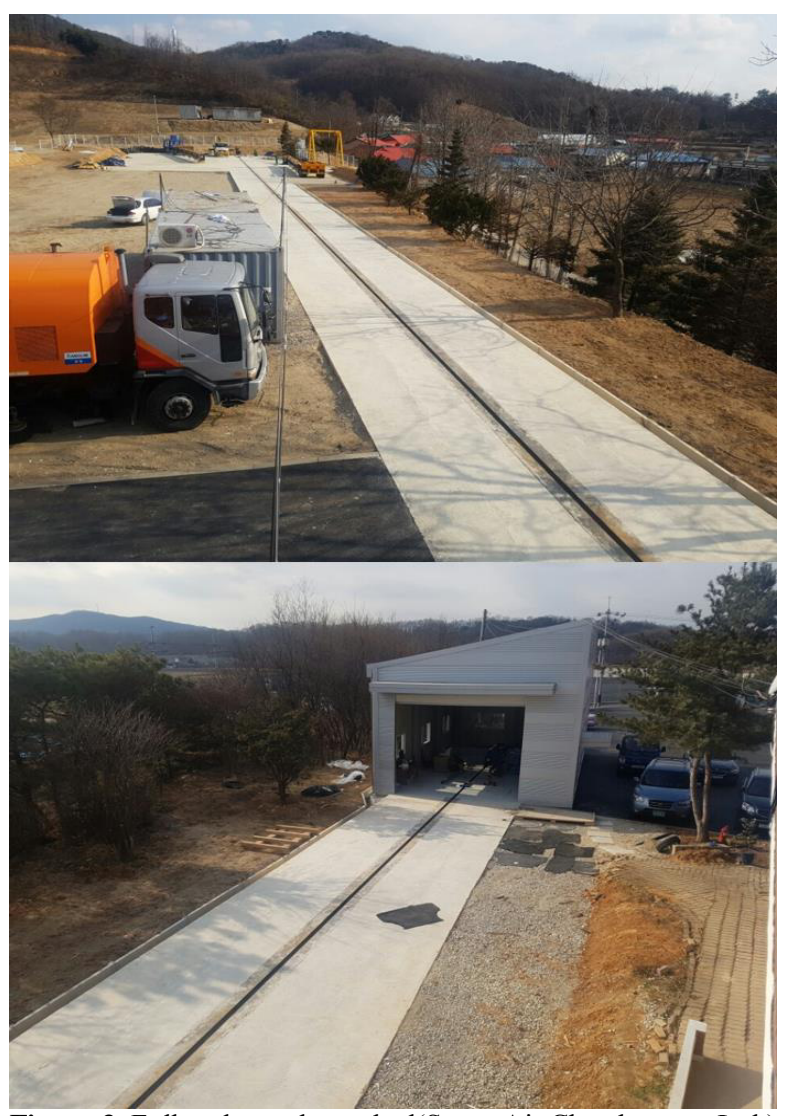

Figure 3. Full scale crash test bed(Smart Air Chamber co., Ltd.)
A length of road test drive road, the maximum driving speed, and the maximum weight of tow truck were $150 \mathrm{~m}$, $120 \mathrm{~km} / \mathrm{h}$, and eight ton, respectively. During the crash test, the program is automatically interlinked thereby collecting THIV, PHD, and ASI values of speed, acceleration, and angular velocity. In addition to the construction, certification of full-scale crash test field that is internationally certified or equivalent level has been planned.

\subsection{Field setting of crash tests}

No crash analysis standards on movable barriers in Korea have been reported. Thus, this study applied and analyzed based on the longitudinal barrier of "Guideline for Sa fety Barriers".

A goal level of large-sized movable barrier through the present study was set to SB2 grade. The crash test was a prototype stage yet so a full-scale crash test was conducted at a level of $90 \%$ prior to cert ification test. The impact speed and angle of the movable barrier were set to $70 \mathrm{~km} / \mathrm{h}$ and 20 degree and an impact vehic le was set to a passenger car( $900 \mathrm{~kg})$.

Table 3 and Figures 4 to 9 present THIV, PHD, and ASI values as results of the full-scale crash test.

The full scale crash test result showed that both types of folder type and telescope type movable barrier satis fied the standard of the passenger safety performance evaluation at a level of $90 \%$. Co mpared to folder type and telescope type, folder type had lower THIV, PHD, ASI than telescope type.

Table 3. Result of occupant protection

\begin{tabular}{|c|c|c|}
\hline Category & Folder type & Telescope type \\
\hline THIV $(\mathrm{km} / \mathrm{h})$ & 15.58 & 22.36 \\
\hline PHD $(\mathrm{g})$ & 2.20 & 13.72 \\
\hline ASI & 0.52 & 1.11 \\
\hline
\end{tabular}

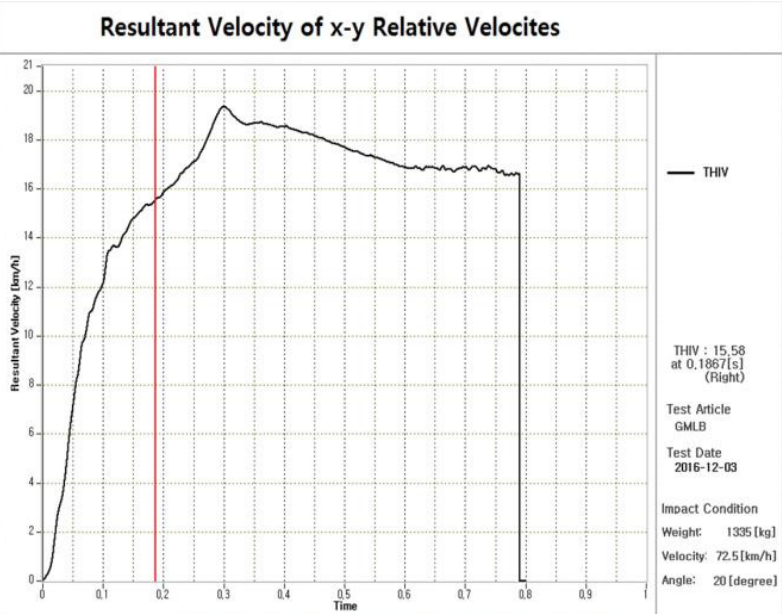

Figure 4. THIV curve of folder type movable barrier 


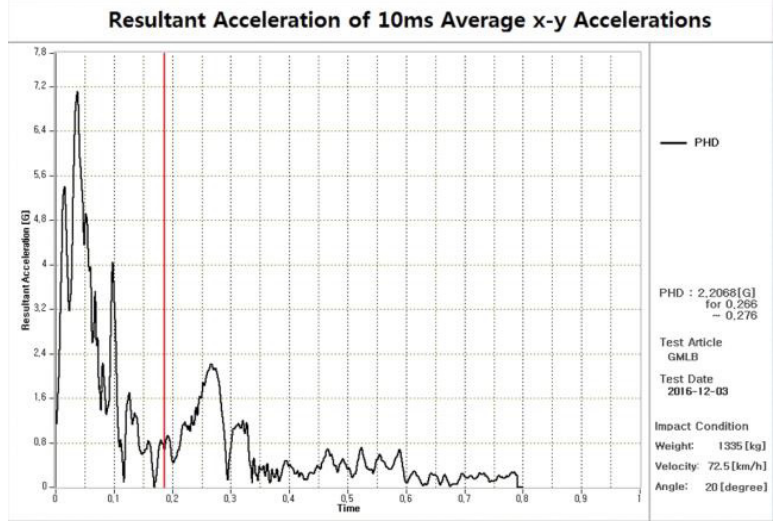

Figure 5. PHD curve of folder ty pe movable barrier

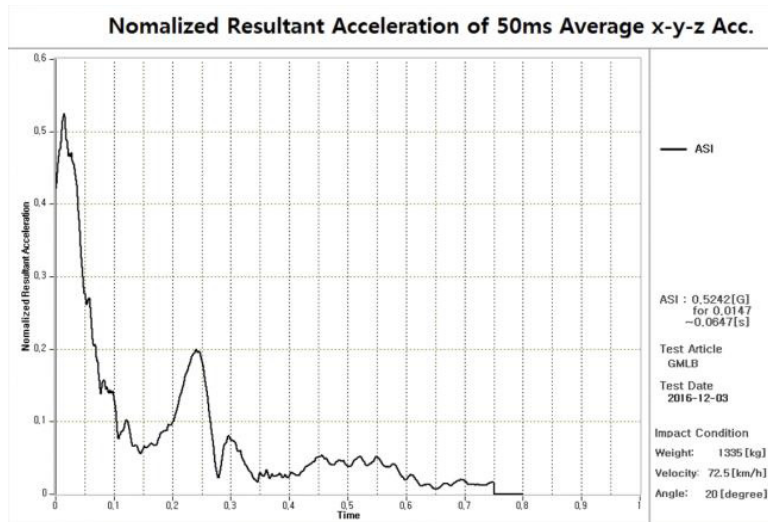

Figure 6. ASI curve of folder type movable barrier

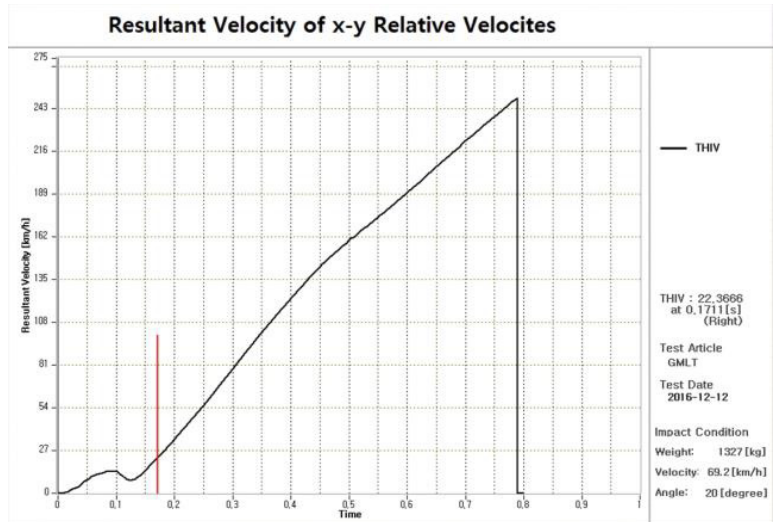

Figure 7. THIV curve of telescope ty pe movable barrier

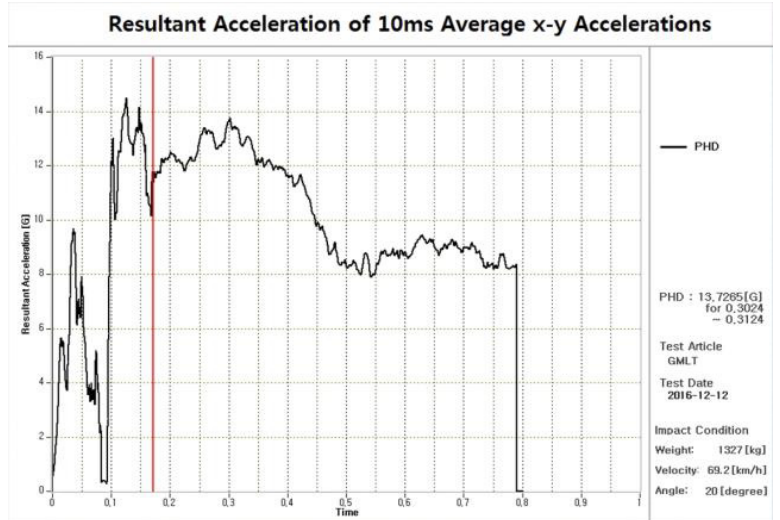

Figure 8. PHD curve of telescope ty pe movable barrier

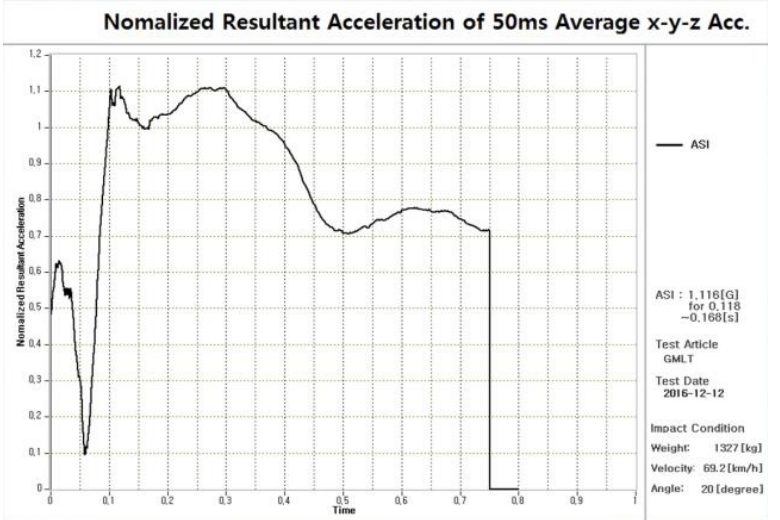

Figure 9. ASI curve of telescope type movable barrier

\section{Conclusion}

This study developed a prototype of large-sized movable barrier and conducted a performance evaluation of occupant protection.

It is significant in that it is first study to carry out the performance evaluation and analysis on the movable barrier which is under development for the safety of road workers in Korea.

The performance of the movable barrier was tested by $900 \mathrm{~kg}$ passenger car, $70 \mathrm{~km} / \mathrm{h}$ impact velocity, 20 degree impact angle. The test procedure was carried out according to the test procedure of "Guideline for Safety Barriers". The conclusions of the present study are as follows:

$\wedge \quad$ The full scale crash test result showed that both types of folder type movable barrier and telescope type movable barrier satisfied the standard of the passenger safety performance evaluation at a level of $90 \%$.

^ The THIV, PHD, ASI value of folder type movable barrier showed that $15.58,2.20,0.52$.

^ The THIV, PHD, ASI value of telescope type movable barrier showed that 22.36, 13.72, 1.11 .

No crash analysis standards on movable barriers in Korea have been reported. Thus, the present study has a limitation that the test results were analyzed based on the longitudinal barrier. And in order to enter the market, it is necessary to consider not only the safety of collision but also the cost of maintenance and manufacturing costs.

In addition, analysis on exit box that analyzes behaviors after crash between a vehicle and large-sized movable barrier should also be conducted.

For the future study, safety performance should be verified through certified authority test after product development to increase a safety level for road workers.

\section{Acknowledgment}

This research was supported by a grant from "Development of Accident Risk Mitigating Technology for Vertical Structure Collision on Road and Road Workers (grant number 17TLRP-C096228-03)" funded by the Ministry of Land, Infrastructure and Transport, Republic of Korea. 


\section{References}

1. AASHTO, Highway Safety Manual (2010)

2. J.G. Kim, C.H. Yang, and D.G. Yoon, "Prioritization of Potential Technology for Establishing a Safe Work Zone Environment", International Journal of Highway Engineering(IJHE), 17(6), 117-126 (2015)

3. Korea Institute of Civil Engineering and Building Technology, Development of Accident Risk Mitigating Technology for Vertical Structure Collision on Road and Road Workers, Internal Document (2015)

4. J.Y. Go, "The Study on Permission and Management System Improvement of Traffic Control System on Road works", Master's Thesis, University of Seoul (2005)
5. M.S. Jin., S.K. Lee, J.P. Moon, Y.S. Kim, and I.S. Park, "Vehicle Based Movable Barriers for Work Zone Safety", Journal of Emerging Trends in Computing and Information sciences(CIS), 7(3), 168-173 (2016)

6. Ministry of Land \& Transportation(MOLIT), Guideline for Safety Barriers (2014)

7. AASHTO, Manual for Assessing Safety Hardware (2009)

8. TRB, NCHRP Report 350:Recommended Procedures for the Safety Performance Evaluation of Highway Features (1993)

9. http://www.dot.ca.gov

10. http:/www.mobilebarriers .com 